\title{
Exclusion of heavy, broad resonances from precise measurements of $W Z$ and $V H$ final states at the LHC
}

\author{
You-Ying Li ${ }^{1}$, Rosy Nicolaidou ${ }^{2}$, Stathes Paganis ${ }^{1, \mathrm{a}}$ (1) \\ ${ }^{1}$ Department of Physics, National Taiwan University, No 1, Sec 4, Roosevelt Road, Taipei 10617, Taiwan \\ ${ }^{2}$ IRFU, CEA, Universite Paris-Saclay, Gif-sur-Yvette, France
}

Received: 8 October 2018 / Accepted: 6 April 2019 / Published online: 17 April 2019

(C) The Author(s) 2019

\begin{abstract}
A novel search for heavy vector resonances in the $H \rightarrow b \bar{b}$ and $Z \rightarrow b \bar{b}$ final states in association with a leptonically decaying $V(Z$ or $W)$ and $W$-only respectively, is proposed. It is argued that excesses with respect to the Standard Model prediction should be observed in all final states $(0,1$ or 2 leptons), with the 1-lepton final state being the strongest. Since the relative strengths of these excesses depend on branching ratios and efficiencies, this is a clear signal for the presence of heavy resonances or their low mass tails. A general vector-triplet model is used to explore the discovery potential as a function of the resonance mass and width. Recent Higgs to $b \bar{b}$ observation data reported by the experiments ATLAS and CMS are used to test the model. Current limits are extended to resonance widths over mass as large as $9 \%$.
\end{abstract}

\section{Introduction}

Heavy vector resonances naturally appear in several extensions of the Standard Model (SM), such as GUT theories [1-3], composite Higgs [4,5], little Higgs [6-8], and models with vector $Z^{\prime}[9,10]$, and $W^{\prime}$ models [11]. The LHC experiments, in most cases, have performed direct searches for heavy narrow resonances decaying to dibosons and $V H$ final states and have put limits to masses up to about $5.5 \mathrm{TeV}$ [12-23].

Broader resonances $(\Gamma / m>4 \%)$ appear for larger values of the resonance coupling strength to weak bosons and the Higgs, $g_{V}$, and depending on their mass, width, and production cross section, the LHC experiments can be sensitive to a large part of the resonance distribution. It is interesting to study to what extent the experiments can be sensitive to the full distribution or tails of such resonances and what the best observables are. In this work, we start addressing this ques- tion with a specific final state: a pair of $b$ quarks produced in association with a weak gauge boson $(Z$ or $W)$ that subsequently decays leptonically (where leptons $\ell$ are electrons and muons). The interest in this final state is because in the large coupling regime, $g_{V}>3$, the heavy boson branching fraction is dominated by decays to $W Z$ and $V H$, while the decays to fermions are suppressed [24]. At the same time, both the Drell-Yan $q \bar{q}$ and the VBF production modes contribute, providing non-negligible cross sections. Here, we propose as an early indirect signal of the presence of vector resonances, the simultaneous excess in the 0,1 , and 2-lepton final states, for both $W Z \rightarrow \ell v b \bar{b}$ and $V H \rightarrow \ell \ell(v) b \bar{b}$, in a measurement of the $b \bar{b}$ quark invariant mass $M_{b b}$. The strength of these excesses in the different final states can be predicted by branching ratios and experimental efficiencies. We argue that the 0,1 , and 2-lepton final states should all be sensitive, albeit with very different sensitivity, to exotic $V^{\prime} \rightarrow V H$ decays. In particular, the 1-lepton state will be the most sensitive in Higgs excesses and in addition, it should also show a smaller level of excess in the $Z$-boson $b \bar{b}$ peak. The pattern of these excesses should be a clear sign of a heavy vector triplet (HVT), even before its observation as a (broad) mass resonance at the $\mathrm{TeV}$ scale. Using a general HVT framework that is also used by the experiments to model such heavy vector triplets [25], we derive the sensitivity of a single LHC experiment as a function of the resonance width.

In this paper, we first introduce the HVT framework and summarize the recent LHC results. In Sect. 3 we present an analysis used to search for heavy vector triplets at the LHC, and we report on the search potential for the $b \bar{b}+0,1$, and 2-lepton final states, for a range of masses and widths. Finally, the compatibility of our predictions with the recently published $13 \mathrm{TeV} H \rightarrow b \bar{b}$ data from ATLAS and CMS in terms of expected and observed limits, is discussed.

\footnotetext{
a e-mail: stathes.paganis@ cern.ch
} 


\section{BSM heavy vector resonances}

A general HVT phenomenological Lagrangian can be used for the modelling of resonances predicted by a wide range of Beyond the Standard Model (BSM) scenarios [25]. The Lagrangian describing the interactions of these resonances $V^{a \prime}, a=1,2,3$ with quarks, leptons, vector bosons and the Higgs boson is shown below:

$$
\begin{aligned}
\mathcal{L}_{V}^{i n t}= & -\frac{g^{2} c_{F}}{g_{V}} V_{\mu}^{a \prime} \bar{q}_{k} \gamma^{\mu} \frac{\sigma_{a}}{2} q_{k}-\frac{g^{2} c_{F}}{g_{V}} V_{\mu}^{a \prime} \bar{\ell}_{k} \gamma^{\mu} \frac{\sigma_{a}}{2} \ell_{k} \\
& -g_{V} c_{H}\left(V_{\mu}^{a \prime} H^{\dagger} \frac{\sigma^{a}}{2} i D^{\mu} H+\mathrm{hc}\right),
\end{aligned}
$$

where $q_{k}$ and $\ell_{k}$ are the quark and lepton weak doublets, $H$ is the Higgs doublet and $\sigma^{a}$ the three Pauli matrices. In this Lagrangian, the HVT triplet $V^{a \prime}=\left(W^{+\prime}, W^{-\prime}, Z^{\prime}\right)$ interacts with the Higgs doublet, i.e. the longitudinal degrees of freedom of the SM $W$ and $Z$ bosons and the SM Higgs, with a coupling strength $g_{V}$. In order to allow for a broader class of models, this coupling strength can be varied by the parameter $c_{H}$, so in the Lagrangian the full coupling to the SM weak and Higgs bosons is $g_{V} c_{H}$. The HVT resonances also couple to the SM fermions, again through their coupling to the SM weak and Higgs bosons, $g^{2} / g_{V}$, where $g$ is the SM $S U(2)_{L}$ weak gauge coupling. This coupling between HVT resonances and fermions is also controlled by an additional parameter $c_{F}$ to allow for a broader range of models to be included, as follows: $g^{2} c_{F} / g_{V}$. As discussed in [23], the experiments consider two Drell-Yan (DY) production scenarios: model $\mathrm{A}$ is a scenario that reproduces the phenomenology of weakly coupled models based on an extended gauge symmetry [9]. In this case, the couplings are $\frac{g^{2} c_{F}}{g_{V}}=-0.55$ and $g_{V} c_{H}=-0.56$, with the fermion coupling being universal. The second DY scenario, referred to as model B, implements a strongly coupled scenario as in composite Higgs models with $\frac{g^{2} c_{F}}{g_{V}}=0.14$ and $g_{V} c_{H}=-2.9$. In model $\mathrm{B}$, the $V^{\prime}$ resonances are broader than in the weakly coupled scenario, model A, but for $\left|g_{V} c_{H}\right| \leq 3$ they remain narrow relative to the experimental resolution. For $\left|g_{V} c_{H}\right|>3$, the resonance intrinsic width becomes significant and cannot be neglected. In summary, the ATLAS and CMS benchmarks correspond for Model A to $c_{H}=-\frac{g^{2}}{g_{V}^{2}}$, $c_{F}=-\frac{1}{3}$ and $g_{V}=1$, and for model B to $c_{H}=-1, c_{F}=1$ and $g_{V}=3$. ATLAS and CMS experimental data from direct $m_{V H}$ and $m_{V V}$ searches, exclude part of the parameter phase space $\left(g_{V}, c_{H}, c_{F}\right)$, for which the intrinsic width $\Gamma$ of the new bosons is dominated by the experimental resolution ( $\sim 4 \%$ of the mass). This is also the case, for example, for $\left|g_{V} c_{H}\right| \leq 3$, where negligible natural width is assumed (narrow width assumption). The goal of this work is to explore the part of the HVT parameter space with $\left|g_{V} c_{H}\right|>3$, where the

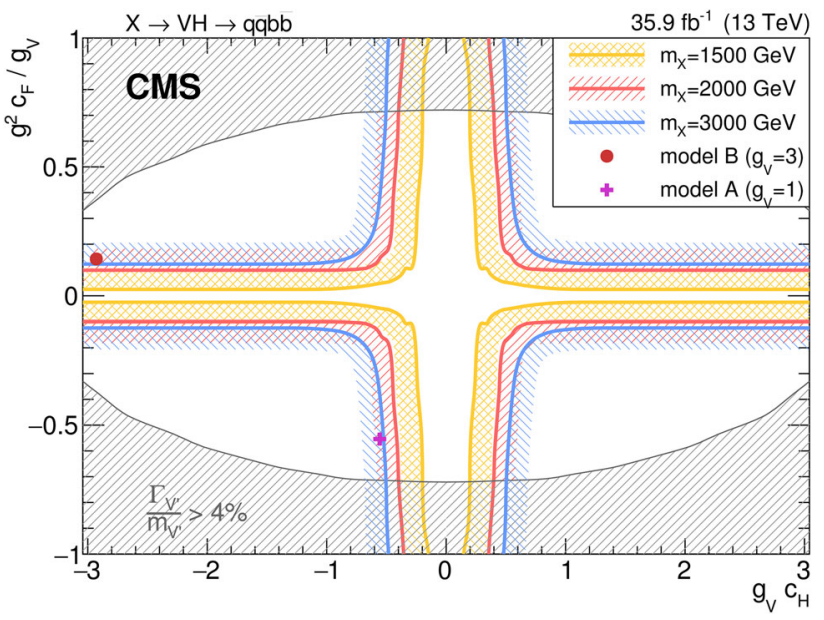

Fig. 1 CMS [21], observed 95\% CL exclusion contours in the HVT parameter space $g_{V} c_{H},\left(g^{2} / g_{V}\right) c_{F}$ for narrow resonances of mass 1.5 TeV, 2.0 TeV and 3.0 TeV. Due to the narrow width approximation assumption, the exclusion validity is restricted to roughly $\left|g_{V} c_{H}\right| \leq 3$ and outside the hatched area shown, thus leaving a large part of the available phase space unconstrained $\left(3<\left|g_{V} c_{H}\right|<4 \pi\right)$. Similar limits have been published by ATLAS [15]

heavy resonances have a significant natural width. Following the model B benchmark, we fix the two constant factors to $c_{H}=-1, c_{F}=1$ and allow $g_{V}$ to vary.

Results from recent experimental analyses that exclude part of the HVT phase space are shown in Fig. 1. The validity of these exclusions is actually limited due to the narrow width approximation. For larger, $\left|g_{V} c_{H}\right|>3$, couplings, the width grows and becomes comparable to or larger than the experimental resolution, leaving a large part of the HVT phase space unexcluded: $3<\left|g_{V} c_{H}\right|<4 \pi$, where $g_{V} \simeq 4 \pi$ is the perturbative limit. This is true even for resonance masses of order $\mathrm{TeV}$.

In this work we argue that the presence of non-zero width resonances, could be observed as an excess of events in the $V H \rightarrow \ell \ell(v) b \bar{b}$ and $W Z \rightarrow \ell v b \bar{b}$ decays with 0,1 , or 2 leptons in the final state. Independent of the fact that due to the experimental resolution the close-by $Z \rightarrow b \bar{b}$ and $H \rightarrow b \bar{b}$ peaks have a partial overlap, in the 1-lepton case the excess must be present in both peaks, while in the 0 and 2-lepton final states it should appear only in the Higgs peak ( $Z^{\prime}$ does not couple to $Z Z$ ). In addition, the excess in the 1-lepton category should be more significant, since in this case both $W^{+}$and $W^{-}$contribute. A combined analysis of the three final states can quantify the excess and correlate it to events with $b \bar{b}$ pairs of higher $p_{T}$ than in the SM Higgs production, since these pairs originate from heavy $\mathrm{TeV}$-scale objects.

ATLAS and CMS recently published results in the search for the SM $H \rightarrow b \bar{b}$ in association with a gauge boson decaying leptonically $[26,27]$. This final state is identical with the one we propose here having sensitivity to HVT resonances. 


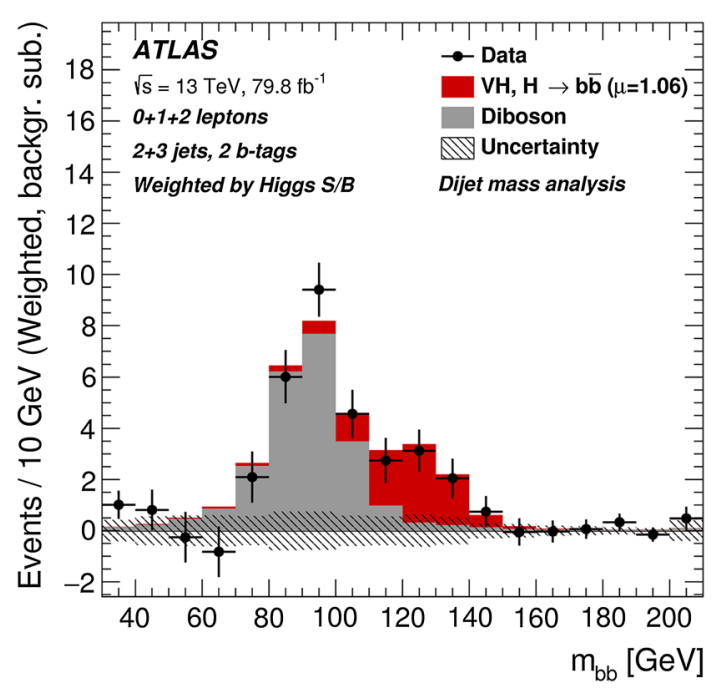

Fig. 2 Weighted bottom anti-bottom quark invariant mass data from ATLAS for $79.8 \mathrm{fb}^{-1}$ [26]. The plot includes all final states with 0,1 and 2 leptons and it compares the data yield with $1.06 \times$ the SM expectation of $H \rightarrow b \bar{b}$, and the SM expectation of $Z \rightarrow b \bar{b}$

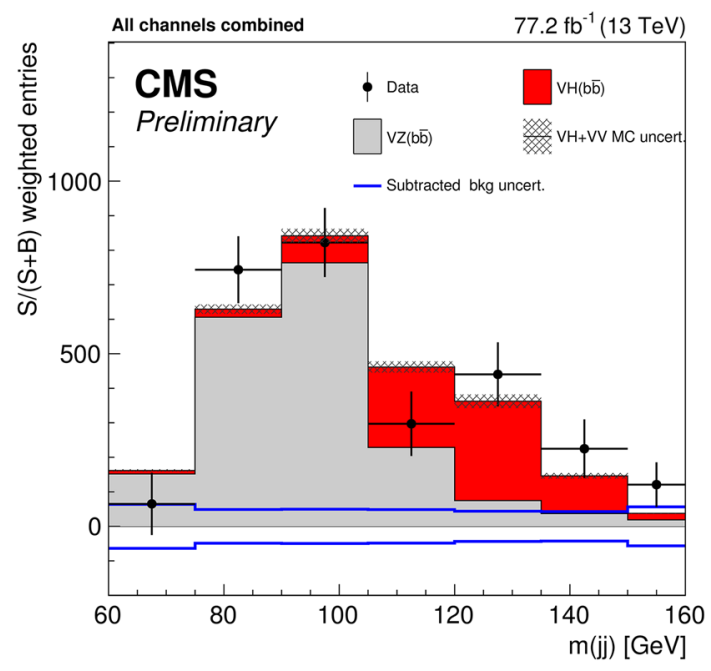

Fig. 3 Weighted bottom anti-bottom quark invariant mass data from CMS for $\sim 77.2 \mathrm{fb}^{-1}$ [27]. The plot includes all final states with 0,1 and 2 leptons and it compares the data yield with the SM expectation of $H \rightarrow b \bar{b}$ and the SM expectation of $Z \rightarrow b \bar{b}$

Both experiments report the measured $M_{b b}$ invariant mass as shown in Figs. 2 and 3, for an integrated luminosity of $\sim 80 \mathrm{fb}^{-1}$ at $13 \mathrm{TeV}$.

Broad heavy resonances mainly decaying to dibosons and pairs of fermions, occur for larger values of the $g_{V}$ coupling $\left(g_{V}>3\right.$ for $c_{H}=-1$ and $\left.c_{F}=1\right)$. The resonance width as a function of $g_{V}$ is shown in Fig. 4 and the branching ratios to a pair of fermions and diboson final states are shown in Fig. 5. In both figures the calculations shown were performed by implementing the HVT model in MadGraph5 [28]. Figure 4 shows that for $g_{V}>3$ the resonance natural width over the mass ratio exceeds $\sim 3 \%$, and as it can be seen in Fig. 5, the

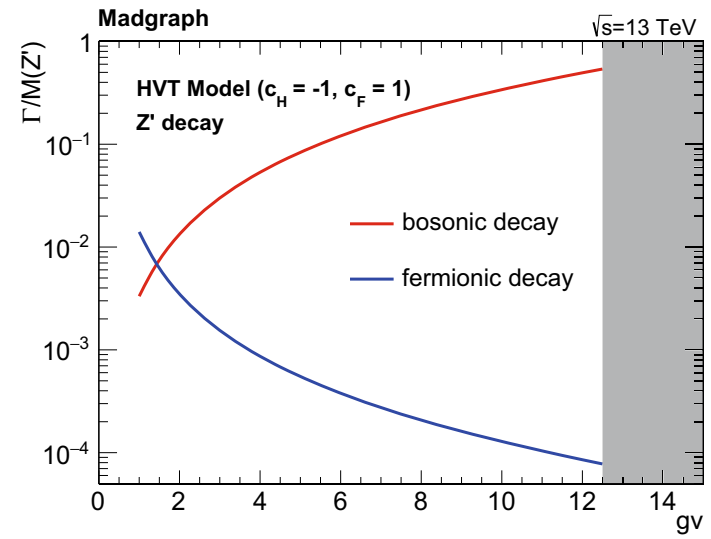

Fig. 4 Heavy resonance width for bosonic and fermionic decays as a function of the $g_{V}$ coupling

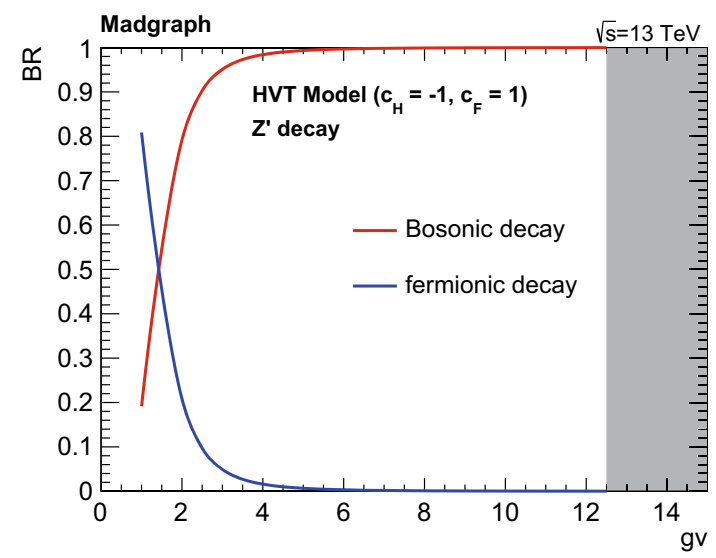

Fig. 5 Heavy resonance branching ratio to bosonic and fermionic decays as a function of the $g_{V}$ coupling

resonance decay to dibosons $(V H, V V)$ is dominant. Direct searches for resonances in the $V V$ and $V H$ channels assume narrow resonance width that corresponds to $g_{V} \leq 3$, leaving unexcluded a large part of the HVT model parameter space.

The magnitude of the impact of these resonances on SM measurements depends on their cross section which in turn, depends on the mass and the $g_{V}$ coupling. Heavy resonance Drell-Yan and Vector Boson Fusion (VBF) production cross sections for masses in the range 1-3 TeV are shown in Fig. 6. The DY mode is always dominant up to large values of $g_{V}$ close to the perturbative limit $g_{V} \simeq 4 \pi$, and the total cross section drops with $g_{V}$. In Fig. 6 we can see that in the mass range $1-2 \mathrm{TeV}$, the cross sections for $g_{V}>3$ range from $O(1)$ to $O(100) \mathrm{fb}$, leading to non-vanishing contributions at present and future measurements. For parts of the parameter space corresponding to low resonance masses and high $g_{V}$ that are theoretically excluded (parts for which the input electroweak parameters $\alpha_{E W}, G_{F}$ and $M_{Z}$, are not reproduced by the HVT model), the cross section is not provided in Fig. 6. 


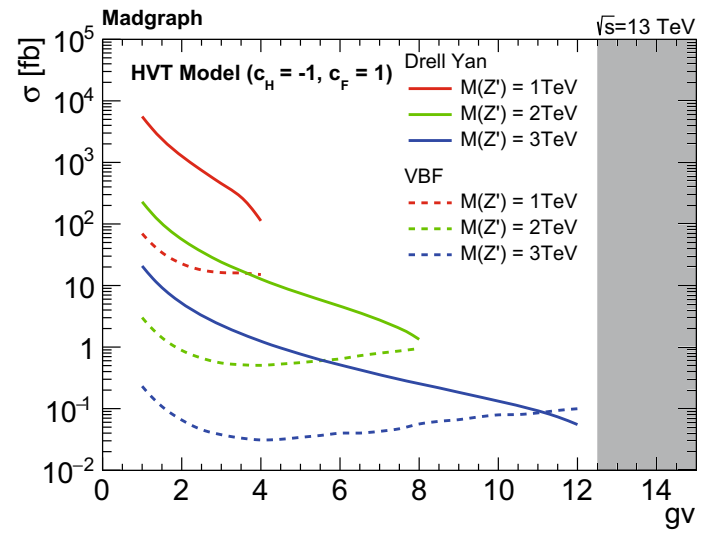

Fig. 6 Heavy resonance cross sections for the DY and VBF production modes, as a function of the $g_{V}$ coupling

\section{Resonance search potential at colliders}

To evaluate the expected signal yields for a broad range of masses and widths, we rely on Monte Carlo samples generated with MadGraph5 [28], interfaced to PYTHIA [29]. To simulate the response of an LHC-like experiment, realistic resolution and reconstruction efficiencies for electrons, muons, photons and jets were applied with the Delphes framework [30]. The specific cut-based event selection was based on the recent $V H$ with $H \rightarrow b \bar{b}$ results from ATLAS and CMS [26,27]. As discussed later, the efficiencies were normalized to the experimental ones. The selected events were split in three categories according to the final state: two $b$ jets with 0,1 or 2 leptons. We follow more closely the ATLAS selection including the requirement of 2 or more jets out of which exactly two must be $b$ jets. We will call this SM selection, the baseline selection, in order to differentiate from additional discriminants which enhance the BSM Higgs signal. The BSM Higgs signal decays to a $b \bar{b}$ pair with a transverse momentum, $p_{\perp}$, significantly larger than the SM Higgs. For this reason, an additional requirement applied to the baseline analysis, is a cut on the transverse momentum of the $b \bar{b}$ system. As in the LHC analyses, we only consider resolved $b \bar{b}$ pairs although the search can be extended to a single, fat $b \bar{b}$ jet analysis. As a discriminant, the $b \bar{b}$ invariant mass $M_{b b}$ is used, which after full selection and subtraction of the $b \bar{b}$ continuum shows two peaks due to the presence of $Z \rightarrow b \bar{b}$ and $H \rightarrow b \bar{b}$.

The characteristic of the BSM signal is that the non-SM produced $H \rightarrow b \bar{b}$ has high $p_{\perp}$. This can be seen in Fig. 7, where the exotic Higgs has typical $p_{\perp}>150 \mathrm{GeV}$ while the SM Higgs is softer. The expected signal and resonant SM background yields for the baseline selection are presented in Table 1 for the 0,1 and 2-lepton final states, for a resonance of mass $1.5 \mathrm{TeV}, g_{V}=5$ and an integrated luminosity of $100 \mathrm{fb}^{-1}$. For a $p_{\perp}>200 \mathrm{GeV}$ cut, we observe a significant

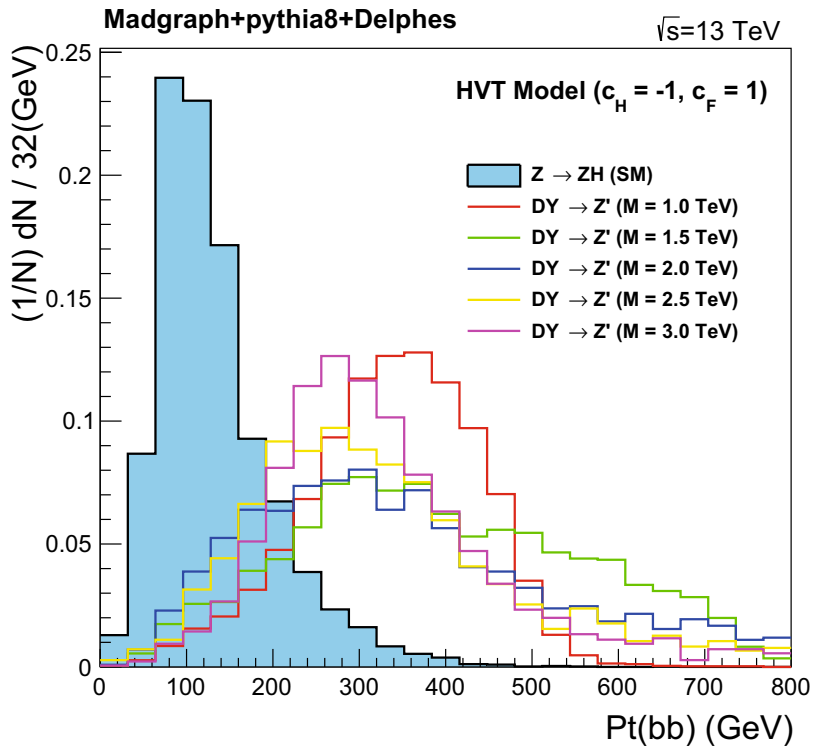

Fig. 7 Exotic Higgs $\rightarrow b \bar{b}$ transverse momentum, $p_{\perp}$, originating from heavy vector resonances, compared with SM Higgs $p_{\perp}$. Exotic Higgs bosons are typically of higher $p_{\perp}\left(p_{\perp}>150 \mathrm{GeV}\right)$

increase in the fraction of BSM signal over the SM resonant yields, that allows to estimate the discovery potential as a function of the HVT model parameters. In the calculations shown in Table 1, in order to realistically estimate the heavy resonance parameter space that can be probed at LHC as a function of luminosity, we have normalized the efficiencies to the published results by the LHC experiments. In this way, we maintain realistic non-resonant background levels and to some extent take systematic differences between the full simulation used by the experiments and the fast simulation used here partially into account. The signal efficiency $x$ acceptance is at the level of few $\%$ and based on the signal cross-section expectations summarized in Fig. 6, observable excesses in $M_{b b}$ should be expected for broad resonance masses below $2 \mathrm{TeV}$, even for present LHC luminosities.

Example scenarios with parameters $M_{Z^{\prime}}=1.5 \mathrm{TeV}$, $g_{V}=4$ and $g_{V}=5$ after baseline selection and a tight transverse momentum cut $p_{\perp}>300 \mathrm{GeV}$, for which the bosonic decay branching ratio is dominant, are presented in Figs. 8 and 9 respectively. In these figures, the combined $M_{b b}$ distribution is shown for an integrated luminosity of $200 \mathrm{fb}^{-1}$. Assuming that the new physics does not modify significantly the SM Higgs couplings, the excess over the SM expectation will indicate the presence of heavy vector resonances decaying into $V H$ and $W Z$ final states. As we have already seen from Table 1, most of the Higgs excess comes from the 1lepton category, while all of the $Z W$ excess comes from the 1-lepton category but it is rather small.

The resonance search potential for a single LHC experiment and an integrated luminosity of $200 \mathrm{fb}^{-1}$, is shown in Figs. 10, 11, and 12, for the 0-lepton, 1-lepton and 2-lepton 
Table 1 Signal yield and resonant $\mathrm{SM}$ background yields for a $1.5 \mathrm{TeV}$ resonance, $g_{V}=5$ and an integrated luminosity of $100 \mathrm{fb}^{-1}$, after baseline selection, and after an additional $p_{\perp}>200 \mathrm{GeV}$ cut (last column). The resonance width over its mass is $\Gamma / m \simeq 10 \%$

\begin{tabular}{lllll}
\hline Process $q q \rightarrow V^{\prime}$ & $\sigma \times B R(\mathrm{fb})$ & $\mathrm{A} \times \epsilon(\%)$ & Yield $\left(100 \mathrm{fb}^{-1}\right)$ & Yield $p_{\perp}>200 \mathrm{GeV}$ \\
\hline$Z^{\prime} \rightarrow Z H \rightarrow \nu \nu b \bar{b}$ & 1.58 & 2.45 & 3.78 & 3.51 \\
$Z \rightarrow Z H \rightarrow \nu \nu b \bar{b}$ & 97.2 & 5.01 & 486 & 224 \\
$Z Z \rightarrow \nu \nu j j$ & 2580 & 0.27 & 697 & 248 \\
$W^{\prime} \rightarrow W H \rightarrow \ell v b \bar{b}$ & 3.87 & 5.5 & 21.3 & 19.4 \\
$W^{\prime} \rightarrow W Z \rightarrow \ell v j j$ & 3.79 & 0.23 & 0.81 & 0.59 \\
$W \rightarrow W H \rightarrow \ell v b \bar{b}$ & 225 & 1.44 & 324 & 148 \\
$W Z \rightarrow \ell \nu j j$ & 4148 & 0.13 & 529 & 173 \\
$Z^{\prime} \rightarrow Z H \rightarrow \ell \ell b \bar{b}$ & 0.553 & 4.76 & 2.6 & 2.2 \\
$Z \rightarrow Z H \rightarrow \ell \ell b \bar{b}$ & 34.2 & 13.7 & 467 & 68.6 \\
$Z Z \rightarrow \ell \ell j j$ & 910 & 0.96 & 875 & 72.6
\end{tabular}

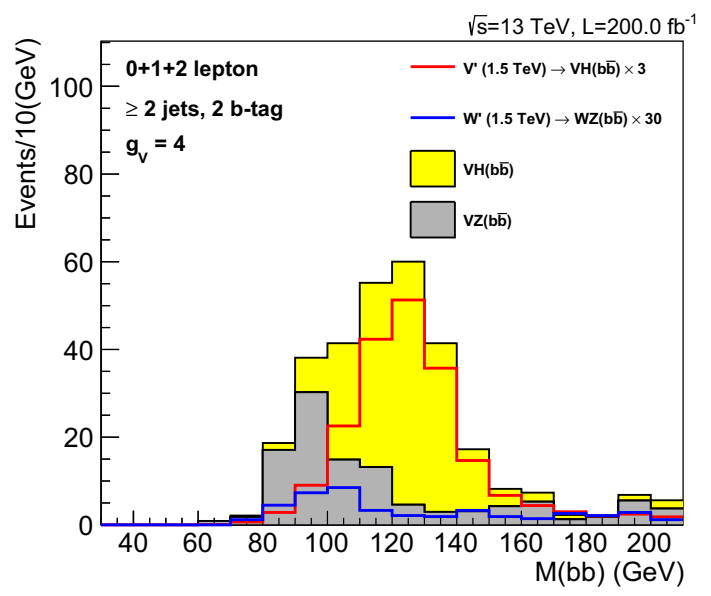

Fig. 8 Reconstructed bottom anti-bottom quark invariant mass for SM $V H \rightarrow \ell(v) \ell(v) b \bar{b}$ and $V Z \rightarrow \ell(v) \ell(v) b \bar{b}$ production (stacked yellow and gray histograms, respectively), for an integrated luminosity of $200 \mathrm{fb}^{-1}$. The HVT model predictions for $M_{V^{\prime}}=1.5 \mathrm{TeV}$ and $g_{V}=4$, with $V H$ shown in red and $W H$ in blue, are overlaid. The plot includes all final states with 0,1 and 2 leptons. $\mathrm{A} p_{\perp}>300 \mathrm{GeV}$ cut has been applied to the $b \bar{b}$ system

categories, respectively [31]. As seen from these results, the search is sensitive to part of the phase space not currently probed by existing searches. For $200 \mathrm{fb}^{-1}$, the sensitivity extends to masses up to about $2 \mathrm{TeV}$, and resonance widths $\Gamma / m \simeq 10 \%$, although the higher the width the lower the resonance mass reach. For $\Gamma / m \simeq 10 \%$ only a mass up to $1.6 \mathrm{TeV}$ can be reached. The reason for the search not being sensitive to larger widths is not only due to the reduction of cross section with $g_{V}$, but also due to a loss of efficiency because for $V^{\prime}$ masses greater than $1.5 \mathrm{GeV}$, the majority of $b$ quark pairs fall in the same jet. More precisely, for resonance masses of $2 \mathrm{TeV}$ or higher, the fraction of resolved $b \bar{b}$ pairs is $5 \%$ or less, respectively. Here it should be stressed that an extension of the proposed search to include fat jets could push these limits at higher values.

In an attempt to test the prediction of the model against the $\sim 80 \mathrm{fb}^{-1}$ data recently reported by ATLAS and CMS,

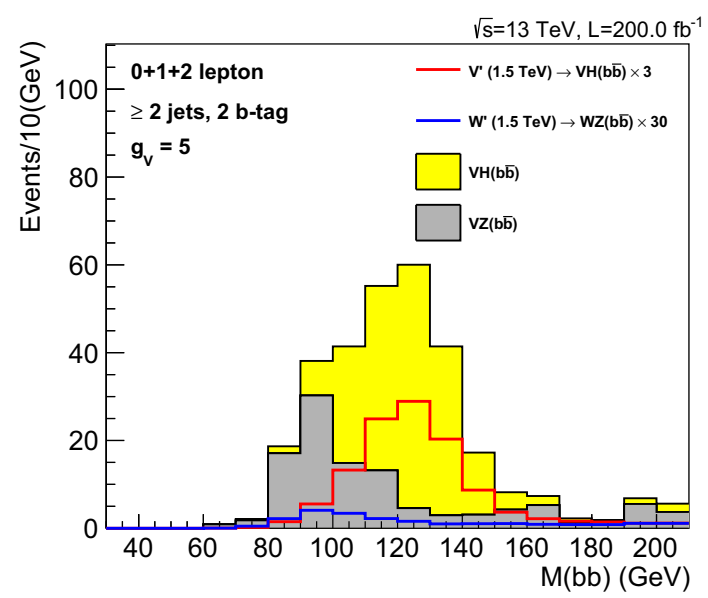

Fig. 9 Reconstructed bottom anti-bottom quark invariant mass for SM $V H \rightarrow \ell(v) \ell(v) b \bar{b}$ and $V Z \rightarrow \ell(v) \ell(v) b \bar{b}$ production (stacked yellow and gray histograms, respectively), for an integrated luminosity of $200 \mathrm{fb}^{-1}$. The HVT model predictions for $M_{V^{\prime}}=1.5 \mathrm{TeV}$ and $g_{V}=5$, with $V H$ shown in red and $W H$ in blue, are overlaid. The plot includes all final states with 0,1 and 2 leptons. A $p_{\perp}>300 \mathrm{GeV}$ cut has been applied to the $b \bar{b}$ system

we estimate the observed limits as shown in Figs. 13, 14, and 15 . The observed limits are based on the signal strength values reported by the experiments in each category. The bands corresponding to the uncertainties in the Higgs signal strength $\mu$ are not shown. The observed limits should be compared to the expected limits with $p_{\perp}>200 \mathrm{GeV}$. As seen in these results, the new LHC data already exclude new parts of the allowed resonance mass-width phase space. For instance from Fig. 14, HVT resonances with $\Gamma / m=9 \%$ and mass up to $1.5 \mathrm{TeV}$, are excluded by the ATLAS experiment. The observed limits in the 1-lepton and 2-lepton categories exclude lower masses and widths than expected because of the corresponding excesses observed by the experiments. However, the uncertainties in each category are still large and more data are needed that will first settle if there is indeed an excess, and then allow to examine the compatibility of 


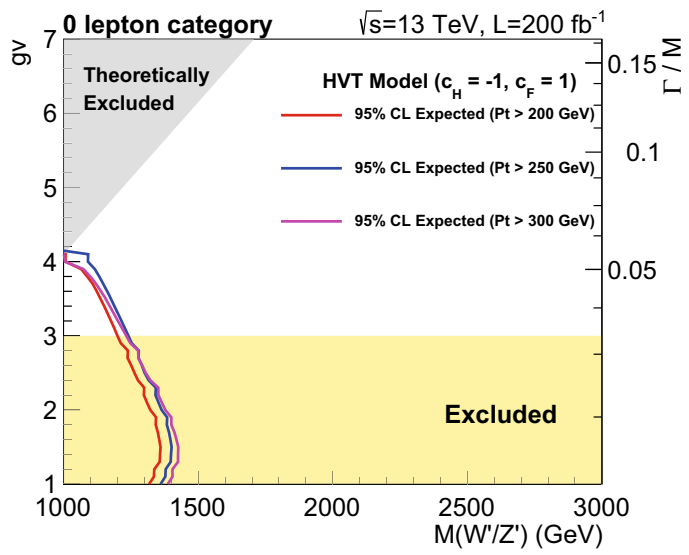

Fig. 10 Expected 95\% CL HVT limits as a function of the resonance mass and width, for an integrated luminosity of $200 \mathrm{fb}^{-1}$. The limits for the 0-lepton category are shown for increasing $p_{\perp}>$ $200,250,300 \mathrm{GeV}$ selections. The theoretically excluded regions correspond to regions of small physical mass and large $g_{V}$ coupling, where the HVT model cannot reproduce the SM input parameters $\alpha_{E W}, G_{F}$ and $M_{Z}$

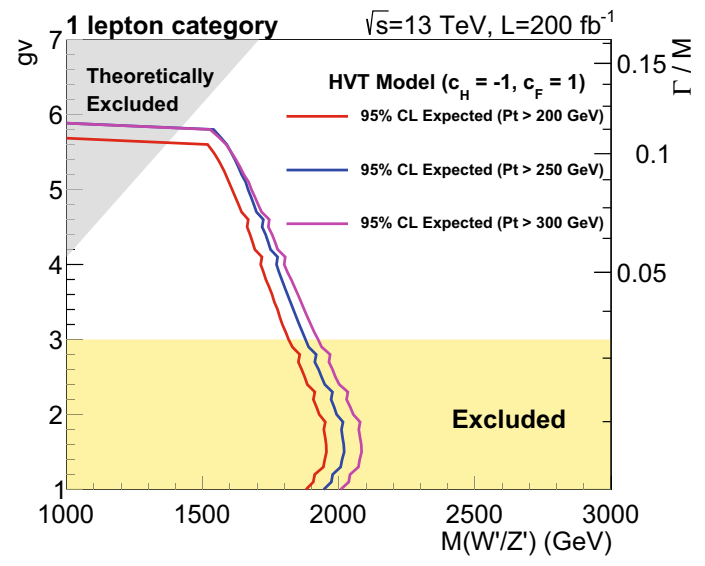

Fig. 11 Expected 95\% CL HVT limits as a function of the resonance mass and width, for an integrated luminosity of $200 \mathrm{fb}^{-1}$. The limits for the 1-lepton category are shown for increasing $p_{\perp}>$ $200,250,300 \mathrm{GeV}$ selections. The theoretically excluded regions correspond to regions of small physical mass and large $g_{V}$ coupling, where the HVT model cannot reproduce the SM input parameters $\alpha_{E W}, G_{F}$ and $M_{Z}$

the pattern of the excess with the HVT model. It should be pointed out that the signal cross-sections were calculated at leading order, thus an additional theory uncertainty due to missing higher order corrections should be considered. These theoretical uncertainties are expected to be small with respect to the rest of the systematic uncertainties of this analysis.

\section{Summary and conclusions}

In this work we explored the potential of a novel search of heavy vector resonances of non-zero natural width, decay-

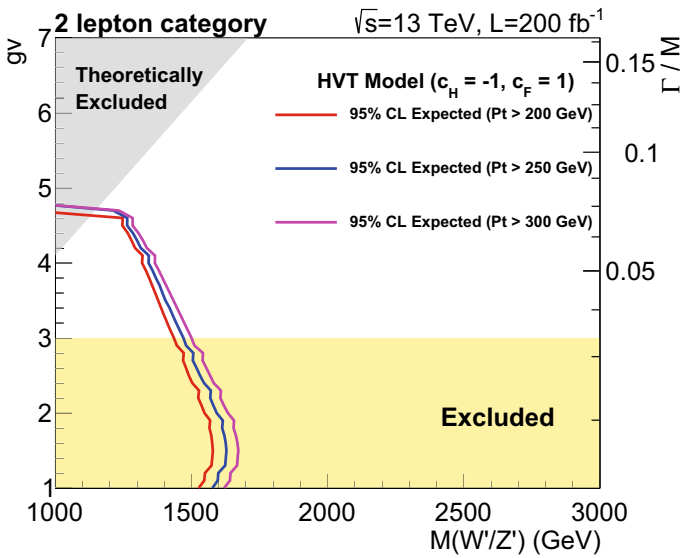

Fig. 12 Expected 95\% CL HVT limits as a function of the resonance mass and width, for an integrated luminosity of $200 \mathrm{fb}^{-1}$. The limits for the 2-lepton category are shown for increasing $p_{\perp}>$ $200,250,300 \mathrm{GeV}$ selections. The theoretically excluded regions correspond to regions of small physical mass and large $g_{V}$ coupling, where the HVT model cannot reproduce the SM input parameters $\alpha_{E W}, G_{F}$ and $M_{Z}$

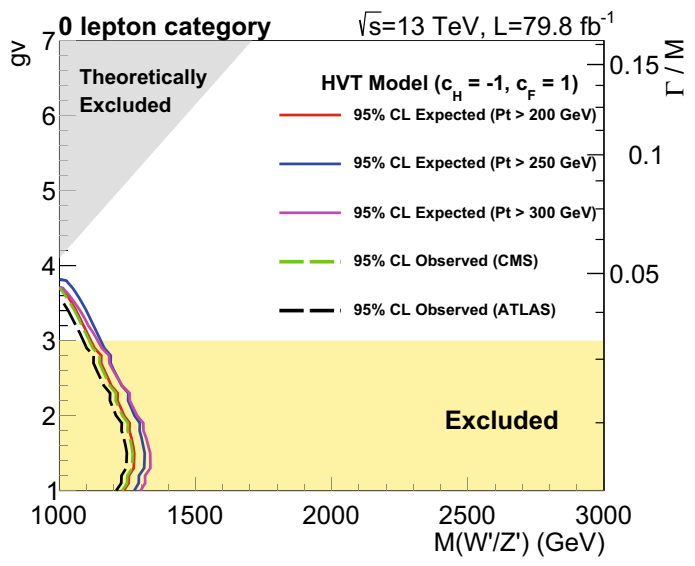

Fig. 13 Expected 95\% CL HVT limits as a function of the resonance mass and width, for an integrated luminosity of $79.8 \mathrm{fb}^{-1}$. The limits for the 0-lepton category are shown for increasing $p_{\perp}>$ 200, 250, $300 \mathrm{GeV}$ selections. Estimated observed limits by ATLAS and CMS are also shown. The theoretically excluded regions correspond to regions of small physical mass and large $g_{V}$ coupling, where the HVT model cannot reproduce the SM input parameters $\alpha_{E W}, G_{F}$ and $M_{Z}$

ing almost exclusively in the $H \rightarrow b \bar{b}$ and $Z \rightarrow b \bar{b}$ final states in association with a leptonically decaying $V$ ( $Z$ or $W)$ and $W$-only, respectively. For large $g_{V}$ coupling of the exotic resonance to the $W$ and $Z$ bosons and the SM Higgs, the branching ratio to $V H$ and to two gauge bosons $V V$ dominates, leading to a simultaneous excess to both Higgs $W H \rightarrow \ell \nu b \bar{b}, Z H \rightarrow \ell \ell b \bar{b}$ and non-Higgs $W Z \rightarrow \ell \nu b \bar{b}$ final states.

We showed that excesses of varying strengths should be observed in all final states ( 0,1 or 2 leptons). The fact that the relative strengths of these excesses depend on branching 


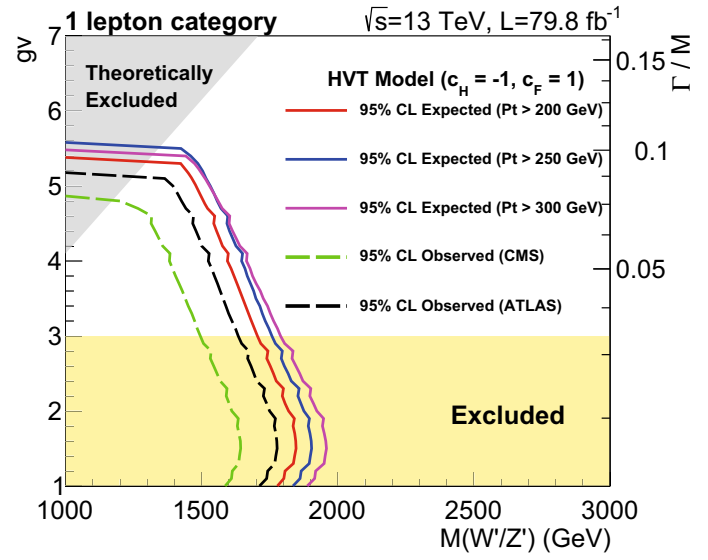

Fig. 14 Expected 95\% CL HVT limits as a function of the resonance mass and width, for an integrated luminosity of $79.8 \mathrm{fb}^{-1}$. The limits for the 1-lepton category are shown for increasing $p_{\perp}>$ 200, 250, $300 \mathrm{GeV}$ selections. Estimated observed limits by ATLAS and CMS are also shown. The theoretically excluded regions correspond to regions of small physical mass and large $g_{V}$ coupling, where the HVT model cannot reproduce the SM input parameters $\alpha_{E W}, G_{F}$ and $M_{Z}$

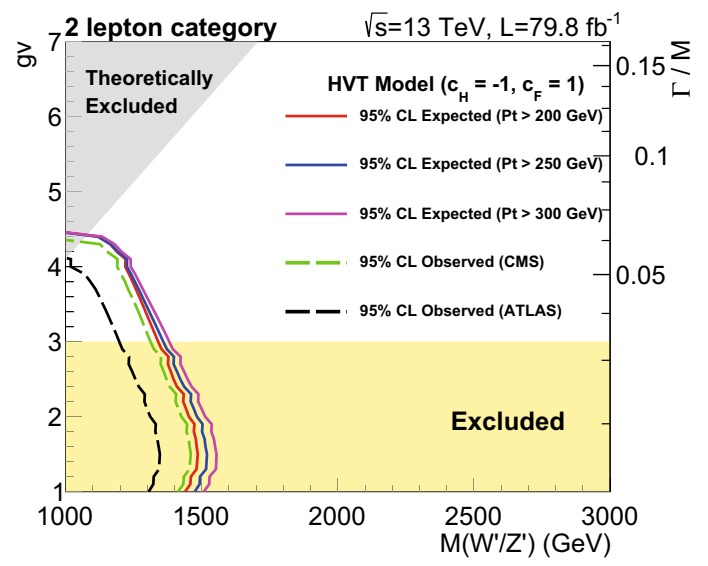

Fig. 15 Expected 95\% CL HVT limits as a function of the resonance mass and width, for an integrated luminosity of $79.8 \mathrm{fb}^{-1}$. The limits for the 2-lepton category are shown for increasing $p_{\perp}>$ 200, 250, $300 \mathrm{GeV}$ selections. Estimated observed limits by ATLAS and CMS are also shown. The theoretically excluded regions correspond to regions of small physical mass and large $g_{V}$ coupling, where the HVT model cannot reproduce the SM input parameters $\alpha_{E W}, G_{F}$ and $M_{Z}$

ratios and efficiencies, provides a clear signature of the presence of heavy resonances or even their low mass tails. For a luminosity accessible by the LHC experiments of $200 \mathrm{fb}^{-1}$, the search is sensitive up to resonance masses of $2 \mathrm{TeV}$ and widths $\Gamma / m$ of $10 \%$. A first test of a heavy vector triplet model against ATLAS and CMS data in terms of expected and observed limits was presented. Although experimental uncertainties on the $V H \rightarrow b \bar{b}$ signal strength $\mu_{V H}$ are still large, the LHC experiments are expected to accumulate significant amounts of data in 2018 and beyond, making the search proposed here a very useful tool in evaluating poten- tial excesses in Higgs yield measurements when the Higgs is produced in association with a weak vector boson.

Acknowledgements This work was supported by the Taiwanese Ministry of Science and Technology under Grant number 106-2112-M-002011-MY3.

Data Availability Statement This manuscript has no associated data or the data will not be deposited. [Authors' comment: The data used to support the findings of this work are available within the article.]

Open Access This article is distributed under the terms of the Creative Commons Attribution 4.0 International License (http://creativecomm ons.org/licenses/by/4.0/), which permits unrestricted use, distribution, and reproduction in any medium, provided you give appropriate credit to the original author(s) and the source, provide a link to the Creative Commons license, and indicate if changes were made. Funded by $\mathrm{SCOAP}^{3}$.

\section{References}

1. J.C. Pati, A. Salam, Lepton number as the fourth color. Phys. Rev. D 10, 275 (1974)

2. H. Georgi, S. Glashow, Unity of all elementary particle forces. Phys. Rev. Lett. 32, 438 (1974)

3. H. Fritzsch, P. Minkowski, Unified interactions of leptons and hadrons. Ann. Phys. 93, 193 (1975)

4. E. Eichten, K. Lane, Low-scale technicolor at the Tevatron and LHC. Phys. Lett. B 669, 235 (2008)

5. R. Contino, The Higgs as a composite Nambu-Goldstone boson (2010). arXiv:1005.4269 [hep-ph]

6. T. Han, H.E. Logan, B. McElrath, L.-T. Wang, Phenomenology of the little Higgs model. Phys. Rev. D 67, 095004 (2003)

7. M. Schmaltz, D. Tucker-Smith, Little Higgs theories. Ann. Rev. Nucl. Part. Sci. 55, 229 (2005)

8. M. Perelstein, Little Higgs models and their phenomenology. Prog. Part. Nucl. Phys. 58, 247 (2007)

9. V.D. Barger, W.-Y. Keung, E. Ma, A gauge model with light $W$ and $Z$ bosons. Phys. Rev. D 22, 727 (1980)

10. E. Salvioni, G. Villadoro, F. Zwirner, Minimal $Z^{\prime}$ models: present bounds and early LHC reach. JHEP 09, 068 (2009)

11. C. Grojean, E. Salvioni, R. Torre, A weakly constrained $W^{\prime}$ at the early LHC. JHEP 07, 002 (2011)

12. ATLAS Collaboration, Search for diboson resonances with bosontagged jets in pp collisions at $\sqrt{s}=13 \mathrm{TeV}$ with the ATLAS detector. Phys. Lett. B 777, 91 (2017)

13. ATLAS Collaboration, Searches for heavy $Z Z$ and $Z W$ resonances in the $\ell \ell q q$ and $v v q q$ final states in pp collisions at $\sqrt{s}=13 \mathrm{TeV}$ with the ATLAS detector. JHEP 03, 009 (2018)

14. ATLAS Collaboration, Search for $W W / W Z$ resonance production in $\ell v q q$ final states in pp collisions at $\sqrt{s}=13 \mathrm{TeV}$ with the ATLAS detector. JHEP 03, 042 (2018)

15. ATLAS Collaboration, Search for heavy resonances decaying into a $W$ or $Z$ boson and a Higgs boson in final states with leptons and b-jets in $36 \mathrm{fb}^{-1}$ of $\sqrt{s}=13 \mathrm{TeV}$ pp collisions with the ATLAS detector. JHEP 03, 174 (2018)

16. ATLAS Collaboration, Search for heavy resonances decaying into a $W$ or $Z$ boson and a Higgs boson in the $q q b b$ final states in pp collisions at $\sqrt{s}=13 \mathrm{TeV}$ with the ATLAS detector. Phys. Lett. B 774, 494 (2017)

17. CMS Collaboration, Search for heavy resonances decaying into a vector boson and a Higgs boson in final states with charged leptons, neutrinos and b quarks at $\sqrt{s}=13 \mathrm{TeV}$. JHEP 11, 172 (2018) 
18. CMS Collaboration, Search for new heavy resonances decaying into a $Z$ boson and a massive vector boson in the $2 \ell 2 q$ final state at $\sqrt{s}=13$ TeV. CMS-PAS-B2G-17-013 (2017)

19. CMS Collaboration, Search for heavy resonances decaying to pairs of vector bosons in the $\ell v q \bar{q}$ final state at $\sqrt{s}=13 \mathrm{TeV}$. CMSPAS-B2G-16-029 (2017)

20. CMS Collaboration, Search for heavy resonances decaying into a $Z$ boson and a vector boson in the $v v q \bar{q}$ final state. CMS-PASB2G-17-005 (2017)

21. CMS Collaboration, Search for heavy resonances that decay into a vector boson and a Higgs boson in hadronic final states at $\sqrt{s}=$ 13 TeV. Eur. Phys. J. C 77(9), 636 (2017)

22. CMS Collaboration, Combination of searches for heavy resonances decaying to $W W, W Z, Z Z, W H$, and $Z H$ boson pairs in protonproton collisions at $\sqrt{s}=8$ and $13 \mathrm{TeV}$. Phys. Lett. B 774, 533 (2017)

23. ATLAS Collaboration, Combination of searches for heavy resonances decaying into bosonic and leptonic final states using $36 \mathrm{fb}^{-1}$ of proton-proton collision data at $\sqrt{s}=13 \mathrm{TeV}$ with the ATLAS detector. Phys. Rev. D 98, 5 (2018)
24. M. Hoffmann, A. Kaminska, R. Nicolaidou, S. Paganis, Probing compositeness with Higgs boson decays at the LHC. Eur. Phys. J. C 74(11), 3181 (2014)

25. D. Pappadopulo, A. Thamm, R. Torre, A. Wulzer, Heavy vector triplets: bridging theory and data. JHEP 09, 060 (2014)

26. ATLAS Collaboration, Observation of $H \rightarrow b b$ decays and $V H$ production with the ATLAS detector. Phys. Lett. B 786, 59 (2018)

27. CMS Collaboration, Observation of Higgs boson decay to bottom quarks. Phys. Rev. Lett. 121, 12 (2018)

28. J. Alwall et al., Madgraph 5: going beyond. JHEP 06, 128 (2011)

29. T. Sjostrand, S. Mrenna, P. Skands, A brief introduction to PYTHIA 8.1. Comput. Phys. Commun. 178, 852 (2008)

30. S. Ovyn, X. Rouby, V. Lemaitre, DELPHES, a framework for fast simulation of a generic collider experiment (2009). arXiv:0903.2225 [hep-ph]

31. G. Cowan, K. Cranmer, E. Gross, O. Vitells, Asymptotic formulae for likelihood-based tests of new physics. Eur. Phys. J. C 71, 1-19 (2011) 